\title{
ANÁLISIS DEL RIESGO BETA EN EL MERCADO BURSÁTIL ESPAÑOL
}

\author{
Rosa María Cáceres Apolinario* \\ Departamento de Economía Financiera y Contabilidad, ULPGC \\ Juan García Boza \\ Departamento de Economía Financiera y Contabilidad, ULPGC
}

(Recibido 3 de diciembre 2003, aceptado 9 de marzo 2004)

\section{Resumen}

El trabajo realiza un análisis del riesgo beta de distintos activos y carteras en el mercado bursátil español durante el período 1991-2000. Se analiza la estabilidad del coeficiente beta a lo largo del tiempo a través de distintos contrastes fundamentados en los residuos recursivos, se estudia su comportamiento temporal mediante estimaciones con distintos intervalos temporales, y también se lleva a cabo la predicción de dicho riesgo a través de diversas metodologías. Para ello, se efectúa la estimación del riesgo beta mediante la técnica de Mínimos Cuadrados Generalizados y se utilizan distintos índices bursátiles como proxys de la cartera de mercado.

\section{Abstract}

The objective of this paper is the analysis of the beta risk of different individual stocks and asset portfolios from the Spanish Stock Market during the period 1991-2000. Analysing the stability of the beta coefficient over time by means of contrasts based on the recursive residuals, studying the temporal behaviour by means of different intervals, and obtaining the forecast by means of different methodologies. To do this, we carry out estimates of the beta risk obtained from Generalised Least Squares, using different stock exchange indexes as proxies of the market portfolio.

Clasificación JEL: G110, G120

Palabras clave: Beta, Estabilidad, Comportamiento temporal, Predicción

* Universidad de Las Palmas de Gran Canaria. Departamento de Economía Financiera y Contabilidad. C/Saulo Torón, 4 Edificio departamental D, 35017, Las Palmas de Gran Canaria. Correo electrónico: rcaceres@defc.ulpgc.es 


\section{Introducción}

Uno de los pilares básicos de la moderna economía financiera, está constituido por los modelos de valoración de activos financieros, destacando entre ellos el Capital Asset Pricing Model (CAPM), desarrollado inicialmente por Sharpe (1964), Lintner (1965) y Mossin (1966) a partir de la Teoría de Selección de Carteras formulada por Markowitz (1952). El modelo CAPM explica las relaciones de equilibrio entre rentabilidad y riesgo, poniéndose de manifiesto que el riesgo beta o riesgo sistemático es el único factor relevante a la hora de determinar la rentabilidad esperada de cualquier activo. En este sentido, el riesgo beta correspondiente a un activo financiero, según el conocido Modelo de Mercado de Sharpe (1963), nos informa de la sensibilidad de las variaciones en la rentabilidad de tal activo a las correspondientes variaciones en la rentabilidad del mercado, pudiéndose clasificar los distintos activos en defensivos, agresivos o neutros, según el riesgo beta sea inferior a la unidad, superior o igual.

En la actualidad el riesgo beta se ha convertido en una importante herramienta para la toma de numerosas decisiones financieras. En este sentido, el coeficiente beta permite desarrollar estrategias de inversión y el costo de capital, analizar coberturas de riesgo con activos derivados, gestionar y evaluar carteras, así como ser de utilidad en cualquier otra modalidad de análisis financiero donde se requiera una medida del riesgo. Teniendo en cuenta la importancia del riesgo beta, es evidente que el simple conocimiento de sus valores históricos, si bien es información relevante, no es suficiente y debe ampliarse con su proyección futura, obteniéndose predicciones o estimaciones futuras de lo que pueda suceder.

No obstante, para la utilización de dicho coeficiente como característico del riesgo de un activo financiero, es preciso analizar su estabilidad y comportamiento temporal, ya que cuanto más elevado sea su grado de estabilidad, mayor confianza se tendría en este coeficiente y por consiguiente, las predicciones realizadas proporcionarán una mayor bondad. Por lo tanto, el presente trabajo tiene como objetivo analizar el riesgo beta de los distintos títulos y carteras de activos que cotizan en el mercado bursátil español durante el período 1991-2000. Con el fin de alcanzar dicho objetivo se realiza un análisis de la estabilidad del riesgo beta a través de distintos contrastes fundamentados en los residuos recursivos, se efectúa un estudio de su comportaniento temporal mediante estimaciones realizadas con intervalos temporales de cinco y dos años de duración, y por último, se lleva a cabo una comparación de la capacidad de predicción de diversas metodologías clásicas, con la finalidad de seleccionar aquella que proporcione mejores estimaciones del riesgo beta para la muestra analizada en el mercado español.

El trabajo se estructura como sigue. En la sección 2 se describe la muestra y los datos utilizados. En la sección 3 se analiza la estabilidad del riesgo beta a lo largo del período muestral. En la sección 4 se estudia el comportamiento temporal de dicho riesgo. En la sección 5 se evalúa la capacidad predictiva de distintas metodologías de predicción del coeficiente beta. Finalmente, en la sección 6 se exponen las conclusiones más relevantes de nuestra investigación. 


\section{La muestra objeto de investigación}

Los datos utilizados en nuestro trabajo de investigación están constituidos por las rentabilidades instantáneas mensuales, ajustadas por dividendos, splits y ampliaciones de capital de acciones, que han cotizado en el mercado bursátil español durante el período comprendido entre enero de 1991 y diciembre de 2000. Con dicha base de datos construimos dos muestras, una de activos individuales y otra de carteras sectoriales. Como cartera de mercado se toma distintas aproximaciones, utilizándose tres índices bursátiles: el Índice General de la Bolsa de Madrid (IGBM), ${ }^{1}$ un índice equiponderado y un índice ponderado por capitalización, construyéndose los dos últimos tanto para la muestra de activos individuales como para la de carteras sectoriales.

Para la selección de ambas muestras, partimos inicialmente de la totalidad de activos que cotizan en el mercado al 31 de diciembre de 2000. La muestra de títulos individuales está constituida por todos los activos que han cotizado en más del $75 \%$ de las sesiones diarias correspondientes a cada uno de los diez años, siendo seleccionados un total de 60 títulos, los cuales se recogen en la tabla I del Anexo. En cambio, a los títulos pertenecientes a la muestra de carteras sectoriales no se les ha exigido ningún requisito de frecuencia de contratación, siendo seleccionados un total de. 141 títulos, asignando cada uno de ellos a uno de los 10 sectores siguientes: ${ }^{2}$ Alimentación, Bancos-Finanzas, CarteraInversión, Comunicación, Construcción, Eléctricas, Metal-Mecánica, PetróleoQuímica, Nuevas Tecnologías, y Otras industrias y Servicios.

El procedimiento utilizado para determinar la rentabilidad instantánea de cada una de las carteras sectoriales se describe a continuación. En primer lugar se calcula para cada uno de los meses la rentabilidad simple de la cartera, como la media aritmética de las rentabilidades de los distintos activos que constituyen la misma. ${ }^{3}$ A continuación, la rentabilidad instantánea de cada cartera, $r_{p t}$, se obtiene a partir de la correspondiente simple, utilizando para ello la siguiente ecuación que recoge la equivalencia financiera entre ambas rentabilidades: $r_{p t}=$ $\log _{e}\left(1+R_{p t}\right)$, siendo $R_{p t}$ la rentabilidad simple de una cartera en el mes $t$. Para la elaboración de las rentabilidades continuas del índice equiponderado y del ponderado se han utilizado las rentabilidades de todos los activos disponibles

1 Forman parte de este índice, todas las empresas que cotizan en la Bolsa de Madrid y cumplan simultáneamente los siguientes criterios de liquidez: a) volumen de contratación sea superior a tres millones de euros; b) rotación superior al 10\% de su capitalización en base anual, teniendo en cuenta para ello tan solo el capital flotante; c) frecuencia de contratación superior al 50\% de las sesiones hábiles. Cabe destacar que el IGBM no está integrado por un número fijo de empresas, sino que su número varia, admitiéndose todas aquellas compañías que cumplan el conjunto de requisitos estipulados y excluyéndose del mismo las que incumplan alguno de ellos.

2 Se ha utilizado la clasificación sectorial establecida por la Bolsa de Madrid vigente al 31 de diciembre de 2000.

3 El motivo de calcular rentabilidades simples de las distintas carteras es debido a que las mismas se obtienen como la suma de las rentabilidades simples de los títulos individuales ponderadas por la participación de cada título en dicha cartera. Sin embargo, esta propiedad no se verifica al utilizar rentabilidades continuas, puesto que no se cumple que el logaritmo de una suma sea la suma de los logaritmos de los sumandos. 
en cada muestra, 60 para la de títulos individuales y 141 para la de carteras. El procedimiento seguido para su cálculo es similar al descrito para las carteras sectoriales.

\section{Estabilidad del riesgo beta}

Como paso previo al análisis de la estabilidad del riesgo beta, vamos a efectuar la estimación del Modelo de Mercado de Sharpe (1963) con la finalidad de cuantificar dicho riesgo en cada uno de los títulos y carteras sectoriales que constituyen la muestra. Para ello es necesario realizar la siguiente regresión. lineal entre las rentabilidades mensuales de cada uno de los activos o carteras y la rentabilidad mensual del índice representativo del mercado:

$$
r_{i t}=\alpha_{i}+\beta_{i} R_{M t}+\varepsilon_{i t},
$$

siendo:

$r_{i t}$ : variable aleatoria representativa de la rentabilidad del activo o cartera $i$ en el período $t$.

$\alpha_{i}$ : parámetro a estimar que recoge la parte de la rentabilidad del activo - cartera $i$ que es independiente del mercado y del conjunto de factores recogidos en la perturbación.

$\beta_{i}$ : parámetro a estimar que mide la relación existente entre las fluctuaciones de la rentabilidad del mercado y las correspondientes al activo o cartera $i$.

$R_{M t}$ : variable aleatoria representativa de la rentabilidad del mercado para el período $t$.

$\varepsilon_{i t}$ : perturbación aleatoria del activo o cartera $i$ en el período $t$.

El coeficiente $\beta_{i}$, que nos indica la sensibilidad de la rentabilidad de un activo a las variaciones de la rentabilidad del mercado, ha sido estimado mediante la técnica de Mínimos Cuadrados Generalizados (MCG), la cual corrige los problemas de autocorrelación y heterocedasticidad de la perturbación aleatoria. ${ }^{4}$

Tabla 1: Riesgo beta de los sectores (1991-2000)

\begin{tabular}{cccc}
\hline & & Índice & Indice \\
Sector & IGBM & Equip. & Pond. \\
\hline Alimentación & 0,8023 & 0,4453 & 0,7465 \\
Bancos-Finanzas & 0,6032 & 0,2765 & 0,5817 \\
Cartera-Inversión & 0,8490 & 0,3487 & 0,7939 \\
Comunicación & 0,5913 & 2,6323 & 2,0497 \\
Construcción & 1,0988 & 0,6067 & 1,0157 \\
Electricas & 0,8044 & 0,3368 & 0,7516 \\
Metal-mecánica & 1,0345 & 0,6479 & 1,0265 \\
Petróleo-Química & 0,9963 & 0,5888 & 0,8985 \\
Otros Indust. y Servicios & 0,9389 & 0,5589 & 0,8542 \\
Nuevas Tecnologías & 1,2638 & 0,7769 & 1,2257 \\
\hline
\end{tabular}

4 Los problemas econométricos de autocorrelación y heterocedasticidad se han detectado a través de los contrastes de Durbin y Watson (1972) y la prueba de White (1980), respectivamente. 
Los resultados obtenidos tras la estimación del Modelo de Mercado utilizando los tres índices bursátiles, se recogen para las 10 carteras sectoriales y para el conjunto de títulos individuales, en la tabla 1 y en la 2 , respectivamente.

Tabla 2: Riesgo beta de los activos (1991-2000)

\begin{tabular}{cccccccc}
\hline & & Índice & Indice & & & Indice & Índice \\
Título & IGBM & Equip. & Pond. & Título & IGBM & Equip. & Pond. \\
\hline ACE & 0,5468 & 0,5507 & 0,6061 & FIL & 0,7925 & 0,9198 & 0,8222 \\
ACS & 1,2602 & 1,5562 & 1,2994 & GAL & 0,1335 & 0,2714 & 0,2075 \\
ACX & 0,9763 & 1,0923 & 1,0077 & HKN & 0,7576 & 1,0023 & 0,8082 \\
AGS & 0,8644 & 0,7965 & 0,8749 & IBE & 0,6877 & 0,5905 & 0,7516 \\
ALB & 0,8571 & 0,7589 & 0,8525 & KOI & 0,3681 & 0,4390 & 0,2932 \\
ALT & 0,7390 & 0,8057 & 0,8074 & MAP & 1,1398 & 1,0409 & 1,1630 \\
AMP & 1,1688 & 1,4371 & 1,1749 & MDF & 0,8757 & 1,1282 & 0,9896 \\
ANA & 1,1714 & 1,3010 & 1,2402 & MVC & 0,8315 & 0,9203 & 0,8641 \\
AND & 0,5501 & 0,5842 & 0,5810 & NMQ & 1,0066 & 1,3710 & 1,0845 \\
ASA & 1,2096 & 1,5024 & 1,1570 & PAS & 0,5845 & 0,5483 & 0,5830 \\
ATL & 0,3096 & 0,3042 & 0,3144 & POP & 0,7334 & 0,7378 & 0,8120 \\
AUM & 0,5045 & 0,4656 & 0,5222 & PSG & 0,8549 & 1,0388 & 0,9077 \\
AZC & 1,4130 & 1,5925 & 1,4709 & PUL & 1,2161 & 1,2945 & 1,2206 \\
AZK & 1,2198 & 1,3689 & 1,2204 & REP & 0,8243 & 0,7845 & 0,9017 \\
BAM & 1,3722 & 1,5880 & 1,3261 & SCH & 1,1683 & 0,9806 & 1,2214 \\
BBVA & 1,1670 & 1,0022 & 1,1750 & SNC & 1,2795 & 1,7266 & 1,2764 \\
BKT & 1,0084 & 0,8996 & 1,0289 & STG & 1,3399 & 1,5575 & 1,3615 \\
BVA & 0,4873 & 0,5168 & 0,4944 & TEF & 1,1062 & 0,8039 & 1,1484 \\
CAN & 0,7007 & 0,7263 & 0,7747 & TFI & 1,1496 & 1,2746 & 1,1172 \\
CAS & 0,1706 & 0,3022 & 0,2319 & TUB & 1,3989 & 1,7137 & 1,3915 \\
CBL & 0,3550 & 0,4399 & 0,4065 & TUD & 1,0158 & 1,3007 & 1,0102 \\
CEP & 0,6148 & 0,6132 & 0,5860 & UNF & 0,8330 & 0,6463 & 0,8396 \\
CPF & 1,0995 & 1,1632 & 1,0660 & URA & 1,3001 & 1,3893 & 1,2641 \\
CRI & 1,0796 & 1,2413 & 1,1008 & URB & 1,1226 & 1,2794 & 1,1284 \\
CTG & 0,8569 & 0,7386 & 0,9170 & VAL & 1,2081 & 1,2939 & 1,2566 \\
DRC & 1,1395 & 1,2058 & 1,1805 & VAS & 0,2745 & 0,3994 & 0,3307 \\
ECR & 1,1331 & 1,5761 & 1,0629 & VDR & 0,9372 & 1,0129 & 0,9443 \\
ELE & 0,7767 & 0,6693 & 0,8485 & VIS & 1,2717 & 1,2803 & 1,2435 \\
ENC & 1,3100 & 1,4600 & 1,2522 & ZOT & 0,6761 & 0,7729 & 0,7207 \\
FCC & 1,0849 & 1,2964 & 1,1694 & ZRG & 0,4450 & 0,5229 & 0,4565 \\
\hline
\end{tabular}

De la observación de ambas tablas se aprecia, con independencia del índice bursátil utilizado, que el número de títulos con betas superiores e inferiores a la unidad es similar, por lo que no existe un predominio del carácter agresivo o defensivo de los distintos activos que cotizan en el mercado español. En cambio, el riesgo beta de los sectores de Construcción, Metal-Mecánica y Nuevas Tecnologías han presentado un carácter agresivo, afectándoles en mayor medida las 
variaciones del mercado. Los restantes sectores presentan un carácter defensivo, siendo menos sensibles a los movimientos de la cartera de mercado. Además de lo expuesto, es preciso señalar que el análisis de la significación estadística del coeficiente beta ha puesto de manifiesto que la rentabilidad del mercado es capaz de explicar las variaciones de rentabilidad de los distintos títulos individuales, independientemente del índice utilizado como proxy de la cartera de mercado (IGBM, índice equiponderado y ponderado por capitalización). Por el contrario, en la muestra de carteras sectoriales no resulta significativa la relación lineal entre la rentabilidad de las carteras y la rentabilidad del índice equiponderado, por lo que no utilizaremos dicho índice bursátil en nuestro análisis del riesgo beta.

Una vez estimado el riesgo beta de cada activo y sector, comprobamos si los coeficientes beta estimados son estables a lo largo del horizonte temporal analizado. Para ello puede utilizarse la prueba de Chow (1960), la cual nuestra la existencia o no de cambio estructural, utilizándose cuando se dispone de información sobre una variación estructural producida en algún momento del horizonte temporal analizado. Sin embargo, otra alternativa para verificar la estabilidad del modelo son los contrastes basados en los residuos recursivos, técnica adecuada cuando se desconoce el período en el que se ha llevado a cabo un cambio estructural. La hipótesis nula de estos contrastes es la homogeneidad temporal del modelo, mientras que la hipótesis alternativa es que se ha producido algún cambio estructural a lo largo del período muestral. Para contrastar las hipótesis anteriores mediante los residuos recursivos pueden utilizarse distintas pruebas como la CUSUM, que se basa en la suma acumulada de los residuos recursivos, la CUSUMQ, que se obticne como la suma acumulada de los cuadrados de los residuos recursivos, así como los contrastes de predicción One-Step Forecast y $N$-step Forecast:

De acuerdo con lo expuesto, el análisis de la estabilidad de los distintos coeficientes beta en el mercado español durante el período 1991-2000 se realiza mediante la estimación recursiva, llevándose a cabo los contrastes mencionados anteriormente mediante la representación gráfica de los residuos recursivos junto con sus bandas de confianza. Los resultados para los títulos individuales, ponen de manifiesto que exclusivamente son estables las betas en 14 de los 60 activos cuando se utiliza el índice equiponderado, disminuyendo a 11 al tomar el IGBM y el índice ponderado por capitalización. En este sentido, es importante destacar la existencia de 5 títulos cuyos coeficientes beta permanecen estables a lo largo de los diez años de estudio, independientemente del índice bursátil utilizado. En cambio, el número de activos con betas inestables asciende a 32 al tomar el índice equiponderado y a 37 cuando se toma el IGBM y el índice ponderado. En los restantes coeficientes beta no se puede rechazar la estabilidad temporal, puesto que los residuos recursivos no se salen de las bandas de confianza, aunque si existen indicios de inestabilidad en algún momento del horizonte temporal.

En cuanto a los resultados de las pruebas efectuadas a las distintas carteras sectoriales, muestran que el riesgo beta se mantiene estable en el período 19912000 únicamente en 3 sectores al tomar el índice ponderado por capitalización y en 2 sectores al utilizar el IGBM, rechazándose la estabilidad de dicho coeficiente en 6 de las 10 carteras sectoriales en cada uno de los mencionados índices de mercado. También se pone de relieve la existencia de indicios de inestabilidad 
del coeficiente beta en un sector, al tomar el IGBM, y en dos sectores, tomando el índice ponderado. Independientemente del índice utilizado como proxy de la cartera de mercado, los sectores cuyo riesgo beta permanece estable a lo largo del período analizado son Alimentación y Metal-Mecánica, mientras que son inestables los coeficientes beta de los sectores de Bancos-Financieras, Comunicación, Eléctricas, Petróleo-Química y Nuevas Tecnologías.

Tenemos pues, que en la mayoría de los coeficientes beta de activos y carteras existe evidencia de inestabilidad en algún momento del horizonte temporal, con independencia del índice de mercado, poniéndose de manifiesto que la agrupación de los activos en carteras sectoriales hace que aumente el porcentaje de betas estables a lo largo del tiempo. También es importante señalar la existencia de un número considerable de títulos y carteras sectoriales en los que no se puede rechazar la estabilidad, aunque si existen indicios de inestabilidad en algún momento del tiempo. Una síntesis del estudio de la estabilidad del riesgo beta en el mercado español, para los distintos activos considerados individualmente y para las carteras sectoriales se expone en la tabla 3.

Tabla 3. Análisis de la estabilidad del riesgo beta

\begin{tabular}{|c|c|c|c|c|c|c|}
\hline & \multicolumn{3}{|c|}{ Títulos Individuales } & \multicolumn{3}{|c|}{ Carteras Sectoriales } \\
\hline & & & Indicios & & & Indicios \\
\hline & Estable & Inestable & Inestabil. & Estable & Inestable & Inestabil. \\
\hline IGBM & $18,3 \%$ & $61,7 \%$ & $20 \%$ & $20 \%$ & $60 \%$ & $20 \%$ \\
\hline 1. Equip. & $23,3 \%$ & $53,3 \%$ & $23,4 \%$ & - & - & - \\
\hline I. Pond. & $18,3 \%$ & $61,7 \%$ & $20 \%$ & $30 \%$ & $60 \%$ & $10 \%$ \\
\hline
\end{tabular}

De acuerdo con los resultados obtenidos podemos afirmar que el riesgo beta de los activos individuales y el de los sectores no permanece estable a lo largo del período 1991-2000. No obstante, es necesario destacar que la utilización del índice equiponderado en la muestra de activos, y del índice ponderado en la muestra de carteras, proporciona un mayor número de estimaciones de betas estables.

\section{Comportamiento temporal de las betas}

Puesto que en el epígrafe anterior se ha verificado la inestabilidad del coeficiente beta, tanto para los títulos considerados individualmente, como para las carteras sectoriales, independientemente del índice utilizado como proxy de la cartera de mercado, procede realizar un análisis de la evolución del riesgo beta a lo largo de tiempo. Por ello, este epígrafe tiene como finalidad el estudio del comportamiento temporal del riesgo beta individual y de las carteras sectoriales, mediante la estimación del mismo, utilizando intervalos temporales solapados y no solapados, de cinco y de dos años de duración, respectivamente.

\subsection{Estimaciones quinquenales}

Tras efectuar las estimaciones de los coeficientes beta, para cada uno de los 60 títulos individuales y para las 10 carteras sectoriales, correspondientes a los 
siguientes subintervalos temporales: 1991-1995, 1992-1996, 1993-1997, 19941998, 1995-1999 y 1996-2000, se realiza una comparación del coeficiente beta correspondiente al período 1991-1995 con el obtenido en la estimación realizada en el período 1996-2000. Con respecto a los títulos individuales se aprecia que gran parte de los coeficientes beta disminuyen en el período más reciente, lo cual pone de manifiesto que en los últimos cinco años la sensibilidad de la rentabilidad de los activos con respecto a los movimientos del mercado ha disminuido con respecto al lustro anterior. En este sentido se observa que el riesgo beta se ve reducido en el $76,6 \%$ de los títulos al tomar el IGBM, disminuyendo dicho porcentaje al $73,3 \%$ y $65 \%$ al utilizar el índice ponderado por capitalización y el equiponderado, respectivamente. En cuanto a las carteras sectoriales y con independencia del índice utilizado como proxy de la cartera de mercado, se observa la misma tendencia de disminución del riesgo beta en el intervalo 1996-2000 con respecto al 1991-1995, a excepción del sector de Nuevas Tecnologías y el de Comunicación. El primero de estos sectores aumenta ligeramente su coeficiente beta al tomar el IGBM, disminuyendo su valor con el índice ponderado. En cambio, el riesgo beta del sector de Comunicación desciende al utilizar el IGBM, incrementándose con el índice ponderado por capitalización.

A continuación se efectúa una comparación del carácter, agresivo o defensivo, del riesgo beta estimado en el periodo global 1991-2000, con el carácter de las estimaciones correspondientes a los mencionados períodos quinquenales. El motivo que justifica la realización de este estudio comparativo es analizar si las variaciones del coeficiente beta a lo largo del tiempo, dado que ya se ha comprobado que no es estable, son capaces de modificar su carácter agresivo o defensivo, o por el contrario, se mantiene que siempre toma valores superiores o inferiores a la unidad. Los resultados de dicha comparativa, por índices bursátiles, se muestran en las tablas 4,5 y 6 .

Al considerar al IGBM como índice de mercado, se observa para la mayoría de los títulos individuales y para los distintos sectores, que el carácter del riesgo beta no permanece constante en las estimaciones quinquenales. Sin embargo, se pone de manifiesto que el $26,6 \%$ de los títulos y el $30 \%$ de los sectores presentan coeficientes beta inferiores a la unidad en las estimaciones realizadas en los seis subperíodos temporales, presentando dichos activos y carteras un comportamiento defensivo. Este comportamiento de los mencionados activos y sectores coincide con el obtenido en la estimación del riesgo beta correspondiente al intervalo temporal global 1991-2000. En el 10\% de los títulos, el comportamiento es agresivo en la totalidad de las estimaciones quinquenales, coincidiendo igualmente con el deducido en el período 1991-2000, al obtener un riesgo beta superior a la unidad. Para los restantes activos (que representan el $63,4 \%$ ) y carteras (representando el $70 \%$ ), el coeficiente beta toma valores superiores e inferiores a la unidad, por lo que no tienen un comportamiento definido a lo largo del tiempo. Lo comentado anteriormente se recoge en la Tabla 4. 
Tabla 4: Comportamiento del carácter del riesgo beta (Índice: IGBM)

\begin{tabular}{|c|c|c|c|}
\hline \multicolumn{4}{|c|}{ Títulos Individuales } \\
\hline & $\begin{array}{c}\text { Defensivos } \\
(\beta<1)\end{array}$ & $\begin{array}{l}\text { Agresivos } \\
(\beta>1)\end{array}$ & Irregular \\
\hline $\begin{array}{c}\text { Estimación global } \\
(1991-2000)\end{array}$ & $51,7 \%$ & $48,3 \%$ & - \\
\hline $\begin{array}{l}\text { Estimación por } \\
\text { subintervalos }\end{array}$ & $26,6 \%$ & $10 \%$ & $63,4 \%$ \\
\hline \multicolumn{4}{|c|}{ Carteras Sectoriales } \\
\hline & $\begin{array}{c}\text { Defensivas } \\
(\beta<1)\end{array}$ & $\begin{array}{l}\text { Agresivas } \\
(\beta>1)\end{array}$ & Irregular \\
\hline $\begin{array}{c}\text { Estimación global } \\
(1991-2000)\end{array}$ & $70 \%$ & $30 \%$ & - \\
\hline $\begin{array}{c}\text { Estimación por } \\
\text { subintervalos }\end{array}$ & $30 \%$ & - & $70 \%$ \\
\hline
\end{tabular}

Si tomamos el índice equiponderado como proxy de la cartera de mercado, las estimaciones del Modelo de Mercado en los distintos subintervalos temporales, muestran que la mayor parte de los coeficientes beta presentan un comportamiento regular. En este sentido, se pone de relieve que el $38,3 \%$ de los títulos presentan un comportamiento defensivo al tener un coeficiente beta inferior a la unidad en cada uno de los períodos considerados, mientras que en el $31,6 \%$ de los títulos, dicho coeficiente toma valores superiores a la unidad en todas las estimaciones realizadas, por lo que su carácter es agresivo. EI comportamiento agresivo o defensivo de los mencionados activos en las distintas estimaciones quinquenales, coincide con el carácter observado cuando se realiza la estimación del riesgo para el período global. En los restantes títulos, que representan el $30,1 \%$ del total, no se puede caracterizar el comportamiento temporal del riesgo beta, puesto que él mismo toma valores superiores e inferiores a la unidad en las distintas estimaciones quinquenales llevadas a cabo a lo largo del tiempo. Lo expuesto se sintetiza en la Tabla 5.

Tabla 5: Comportamiento del carácter del riesgo beta (Índice: Equiponderado)

\begin{tabular}{cccc}
\hline & \multicolumn{3}{c}{ Títulos Individuales } \\
\hline & $\begin{array}{c}\text { Defensivos } \\
(\beta<1)\end{array}$ & $\begin{array}{c}\text { Agresivos } \\
(\beta>1)\end{array}$ & Irregular \\
\hline $\begin{array}{c}\text { Estimación global } \\
(1991-2000)\end{array}$ & $48,3 \%$ & $51,7 \%$ & - \\
\hline $\begin{array}{c}\text { Estimación por } \\
\text { subintervalos }\end{array}$ & $38,3 \%$ & $31,6 \%$ & $30,1 \%$ \\
\hline
\end{tabular}


Por último, y considerando a la rentabilidad del índice ponderado por capitalización bursátil como variable exógena en la estimación del riesgo beta, se obtienen coeficientes beta que en su mayoría tienen un comportamiento irregular. Se observa que el $30 \%$ de los activos y el $10 \%$ de las carteras sectoriales presentan un riesgo beta cuyo comportamiento es siempre defensivo en los distintos subperíodos temporales, al tener coeficientes inferiores a la unidad. En cambio, el $11,6 \%$ de los títulos tiene un comportamiento agresivo que se pone de manifiesto en las distintas estimaciones quinquenales del riesgo beta. En ambos casos, el comportamiento experimentado por el riesgo beta en los subperíodos temporales es idéntico al obtenido en el período total 1991-2000. Con respecto a las carteras sectoriales, es importante señalar que ninguna de ellas ha presentado un comportamiento agresivo en todos los subperíodos, a pesar de que el riesgo beta estimado en el período global es superior a la umidad en el $40 \%$ de los sectores. En cuanto a los restantes títulos (que representan el 58,4\% del total) y carteras sectoriales (representando el 90\%) se caracterizan por tomar valores superiores e inferiores a la unidad en las distintas estimaciones del coeficiente beta, por lo que no presentan un comportamiento estable del carácter del riesgo beta a lo largo del tiempo. Lo expuesto se recoge en la Tabla 6 .

Tabla 6: Comportamiento del carácter del riesgo beta (Índice: Ponderado)

\begin{tabular}{cccc}
\hline \multicolumn{4}{c}{ Títulos Individuales } \\
\hline & $\begin{array}{c}\text { Defensivos } \\
(\beta<1)\end{array}$ & $\begin{array}{c}\text { Agresivos } \\
(\beta>1)\end{array}$ & Irregular \\
\hline $\begin{array}{c}\text { Estimación global } \\
(1991-2000)\end{array}$ & $50 \%$ & $50 \%$ & - \\
\hline $\begin{array}{c}\text { Estimación por } \\
\text { subintervalos }\end{array}$ & $30 \%$ & $11,6 \%$ & $58,4 \%$ \\
\hline \multicolumn{4}{c}{ Carteras Sectoriales } \\
\hline & $\begin{array}{c}\text { Defensivas } \\
(\beta<1)\end{array}$ & $\begin{array}{c}\text { Agresivas } \\
(\beta>1)\end{array}$ & Irregular \\
\hline $\begin{array}{c}\text { Estimación global } \\
(1991-2000)\end{array}$ & $60 \%$ & $40 \%$ & - \\
\hline $\begin{array}{c}\text { Estimación por } \\
\text { subintervalos }\end{array}$ & $10 \%$ & - & $90 \%$ \\
\hline
\end{tabular}

\subsection{Estimaciones bienales e interrelación entre el riesgo beta y la pri- ma de riesgo del mercado}

Con la finalidad de estudiar con mayor profundidad el comportamiento temporal del riesgo beta, se ha procedido a dividir el horizonte temporal en cinco subperíodos de 24 meses: 1991-1992, 1993-1994, 1995-1996, 1997-1998 y 19992000; con el propósito de obtener las estimaciones bienales de los distintos activos individuales y de las carteras sectoriales. Posteriormente, se han obtenido 
las diferencias entre las betas de dos subperíodos consecutivos para cada título y sector, y de esta forma observar mejor la evolución temporal del riesgo beta. Así una diferencia positiva indicaría que ha aumentado el riesgo beta, mientras que una diferencia negativa señalaría una disminución de dicho riesgo. Una síntesis de los resultados obtenidos se encuentra en la Tabla 7 , donde se recogen el porcentaje de activos y carteras sectoriales que experimentan variaciones positivas y negativas en el riesgo beta.

Tabla 7: Variaciones en el riesgo beta

\begin{tabular}{|c|c|c|c|c|}
\hline & \multicolumn{4}{|c|}{ Títulos Individuales } \\
\hline & 1993-1994 & $1995-1996$ & $1997-1998$ & $1999-2000$ \\
\hline & 1991-1992 & 1993-1994 & $1995-1996$ & 1997-1998 \\
\hline & $+\quad-$ & $+\quad-$ & $+\quad-$ & $+\quad-$ \\
\hline IGBM & $33,4 \% 66,6 \%$ & $48,4 \% 51,6 \%$ & $40 \% 60 \%$ & $18,4 \% 81,6 \%$ \\
\hline I. Equi. & $46,7 \% 53,3 \%$ & $53,4 \% 46,6 \%$ & $46,7 \% 53,3 \%$ & $43,4 \% 56,6 \%$ \\
\hline \multirow[t]{5}{*}{ I. Pon. } & $36,7 \% \quad 63,3 \%$ & $41,6 \% 58,3 \%$ & $46,7 \% \quad 53,3 \%$ & $20 \% 80 \%$ \\
\hline & \multicolumn{4}{|c|}{ Carteras Sectoriales } \\
\hline & 1993-1994 & 1995-1996 & $1997-1998$ & $1999-2000$ \\
\hline & 1991-1992 & 1993-1994 & $1995-1996$ & $1997-1998$ \\
\hline & & & $+\quad-$ & +- \\
\hline IGBM & $30 \% 70 \%$ & $40 \% 60 \%$ & $50 \% 50 \%$ & $40 \% 60 \%$ \\
\hline I. Pon. & $30 \% 70 \%$ & $20 \% 80 \%$ & $40 \% 60 \%$ & $10 \% 90 \%$ \\
\hline
\end{tabular}

Al comparar para un mismo activo o cartera las variaciones del riesgo beta en los distintos subperíodos temporales, se observa que dichas variaciones no presentan idéntico signo, ya sea positivo o negativo, lo cual nos está indicando la inexistencia de una evolución conjunta del riesgo beta para la totalidad de los activos y carteras sectoriales a lo largo del tiempo. No obstante, se puede afirmar que los distintos títulos y sectores han experimentado variaciones mayoritariamente negativas, lo cual pone de manifiesto un cierto descenso del riesgo beta a lo largo de los períodos bienales analizados.

Además de lo expuesto, se observa para el conjunto de títulos considerados individualmente, que al tomar como índice representativo del mercado el IGBM y el ponderado por capitalización bursátil se obtiene un mayor porcentaje de variaciones negativas, mientras que al utilizar el índice equiponderado en la muestra de títulos individuales, se aprecia la existencia de un cierto equilibrio en los cambios del riesgo beta, puesto que aproximadamente la mitad de las diferencias son positivas y el resto negativas. Con respecto a los distintos sectores, hay que señalar que la mayor proporción de variaciones negativas se obtiene con el índice ponderado. Por último, es importante destacar que, con independencia del índice de mercado utilizado, el sector Construcción ha experimentado una continua disminución del riesgo beta, ya que sus variaciones han sido siempre negativas, y que los sectores de Metal-Mecánica y PetróleoQuímica también han sufrido sucesivas reducciones del riesgo beta, a excepción del aumento producido entre 1997-1998 y 1995-1996. Una situación similar fue 
soportada por el sector de Alimentación, al presentar descensos del riesgo beta, a excepción del incremento producido entre 1995-1996 y 1993-1994.

Cabe preguntarse si la evolución descrita para el riesgo beta de los distintos activos y carteras sectoriales pudiera guardar alguna relación funcional con variables económicas conocidas. Por ello, vamos a determinar la prima de riesgo del mercado bursátil español en cada uno de los subintervalos temporales descritos, con el objetivo de verificar si existe alguna relación entre las variaciones del riesgo beta y la situación de la economía o momento bursátil, medida a través de la prima de riesgo del mercado. Para el cálculo de la misma se ha utilizado la rentabilidad de los distintos índices bursátiles utilizados en la muestra de títulos y de carteras sectoriales, y se ha tomado como rentabilidad del activo libre de riesgo, la correspondiente a las letras del tesoro de 1-3 meses. Los resultados se exponen en la Tabla 8.

Tabla 8: Prima de riesgo del mercado

\begin{tabular}{cccccc}
\hline & $91-92$ & $93-94$ & $95-96$ & $97-98$ & $99-00$ \\
\hline IGBM & $-1,19 \%$ & $0,41 \%$ & $1,19 \%$ & $2,40 \%$ & $-0,22 \%$ \\
I. Ponderado (60 tit.) & $-0,25 \%$ & $0,84 \%$ & $2,00 \%$ & $2,59 \%$ & $0,10 \%$ \\
I. Equiponderado (60 tit.) & $-1,55 \%$ & $1,66 \%$ & $0,98 \%$ & $2,88 \%$ & $-0,90 \%$ \\
I. Ponderado (141 tit.) & $-0,48 \%$ & $0,88 \%$ & $1,85 \%$ & $2,06 \%$ & $-0,09 \%$ \\
\hline
\end{tabular}

Tal y como se observa en la anterior tabla, independientemente del índice utilizado, la prima de riesgo del mercado bursátil español es positiva en los subperíodos 1993-1994, 1995-1996 y 1997-1998, por lo que podemos considerar que el mercado está en alza, de manera que los inversores han sido retribuidos por el riesgo asumido al invertir en activos de renta variable. En este sentido, hay que destacar el incremento experimentado por la prima de riesgo en 1997-1998 con respecto a los anteriores subperíodos. Por el contrario, en el intervalo 1991-1992 dicha prima resulta ser negativa, situándose el mercado español a la baja, no retribuyéndose a los inversores el riesgo asumido por la renta variable, por lo que resulta más rentable invertir en el activo libre de riesgo. En el último intervalo temporal 1999-2000, vamos a considerar de forma general que el mercado se encuentra a la baja, a pesar de que existan diferencias en función del índice utilizado. ${ }^{5}$

Con la finalidad de verificar los cambios experimentados en la prima de riesgo del mercado bursátil español, se recoge en la Tabla 9 las diferencias de la misma en dos períodos de tiempo consecutivos.

5 En este sentido, se observa que la prima de riesgo es negativa al tomar el IGBM, el equiponderado con 60 títulos y el índice ponderado por capitalización con 141 títulos, siendo positiva exclusivamente con el índice ponderado con 60 títulos. 
Tabla 9: Diferencia de la prima de riesgo del mercado

\begin{tabular}{ccccc}
\hline & $1993-1994$ & $1995-1996$ & $1997-1998$ & $1999-2000$ \\
& $1991-1992$ & $1993-1994$ & $1995-1996$ & $1997-1998$ \\
\hline IGBM & $1,60 \%$ & $0,78 \%$ & $1,21 \%$ & $-2,62 \%$ \\
I. Ponde. (60 tit.) & $1,09 \%$ & $1,16 \%$ & $0,59 \%$ & $-2,49 \%$ \\
I. Equip. (60 tit.) & $3,21 \%$ & $-0,68 \%$ & $1,90 \%$ & $-3,78 \%$ \\
I. Ponde. (141 tit.) & $1,36 \%$ & $0,97 \%$ & $0,21 \%$ & $-2,15 \%$ \\
\hline
\end{tabular}

En cuanto a las variaciones sufridas por la prima de riesgo del mercado, podemos afirmar que en la casi totalidad de los subperíodos, el comportamiento de la misma ha sido idéntico con los distintos índices de mercado utilizados, excepto al comparar el período 1995-1996 con el 1993-1994, ya que al tomar el índice equiponderado con 60 títulos, se produce una disminución de la prima de riesgo, mientras que con los restantes índices existe un incremento de la misma. En cualquier caso, y para nuestro análisis, vamos a considerar que se ha producido un aumento de la prima de riesgo. Con respecto a la evolución del riesgo beta, indicar que se ha producido un crecimiento del mismo hasta 1997-1998, decreciendo en 1999-2000, poniéndose de manifiesto que la prima de riesgo en el mercado bursátil es variable durante el período temporal analizado.

La comparación de las diferencias entre los coeficientes beta de los títulos individuales y la situación del mercado bursátil, tal y como se recoge en la Tabla 10 , nos muestra la existencia de un porcentaje considerable de títulos cuyos cambios en el riesgo beta coinciden con las variaciones experimentadas por la prima de riesgo del mercado en cada uno de los subperíodos, fundamentalmente entre 1999-2000 y 1997-1998. Es decir, que los coeficientes beta de dichos valores disminuyen cuando lo hace la prima de riesgo del mercado, mientras que aumentan su riesgo beta al incrementarse la prima de riesgo del mercado, existiendo un relación positiva entre la beta y la situación de la economía.

Table 10: Títulos con variaciones en el riesgo beta similar a la prima de riesgo del mercado

\begin{tabular}{lcccc}
\hline & $1993-1994$ & $1995-1996$ & $1997-1998$ & $1999-2000$ \\
& $1991-1992$ & $1993-1994$ & $1995-1996$ & $1997-1998$ \\
\hline IGBM & $33,4 \%$ & $48,4 \%$ & $40 \%$ & $81,6 \%$ \\
I. Equiponderado & $46,6 \%$ & $53,4 \%$ & $46,7 \%$ & $56,6 \%$ \\
I. Ponderado & $36,6 \%$ & $41,6 \%$ & $46,7 \%$ & $80 \%$ \\
\hline
\end{tabular}

Al observar en la anterior tabla las diferencias del riesgo beta del intervalo 1999-2000 con respecto al 1997-1998, considerando como proxy de la cartera de mercado el índice IGBM, se obtiene un mayor número de títulos cuyas variaciones en el riesgo beta se mueven simultáneamente con la prima de riesgo del mercado. Por el contrario, en los restantes subintervalos, el índice equiponderado es el que proporciona un mayor porcentaje de títulos con movimientos del coeficiente beta similar a la prima de riesgo del mercado. 
Si comparamos las diferencias del riesgo beta de las distintas carteras sectoriales con la situación de la economía o del mercado bursátil, tal y como se recoge en la Tabla 11, se observa cómo diversos sectores presentan variaciones del riesgo beta similar a la prima de riesgo del mercado, coincidiendo fundamentalmente dichos cambios entre 1999-2000 y 1997-1998. Se observa también, independientemente del índice utilizado como proxy del mercado, que con el paso del tiempo aumenta el porcentaje de sectores cuyas variaciones del riesgo beta se mueven simultáneamente con la prima de riesgo del mercado.

Tabla 11: Carteras sectoriales con variaciones similares a la prima de riesgo del mercado

\begin{tabular}{ccccc} 
& $1993-1994$ & $1995-1996$ & $1997-1998$ & $1999-2000$ \\
& $1991-1992$ & $1993-1994$ & $1995-1996$ & $1997-1998$ \\
\hline IGBM & $30 \%$ & $40 \%$ & $50 \%$ & $60 \%$ \\
I. Ponderado & $30 \%$ & $20 \%$ & $40 \%$ & $90 \%$ \\
\hline
\end{tabular}

En relación a la cartera de mercado, se aprecia que en el intervalo 1999-2000 con respecto al 1997-1998, el índice ponderado por capitalización es el que presenta un mayor número de sectores cuyas variaciones del riesgo beta se mueven simultáneamente con la prima de mercado. En cambio, en las restantes diferencias del riesgo beta, al tomar el índice ponderado por capitalización, se obtiene un porcentaje más elevado de carteras sectoriales con variaciones idénticas a la situación de la economía.

Tal y como se ha expuesto, existen sectores y activos cuyas variaciones en el riesgo beta coinciden con las experimentadas por la prima de riesgo del mercado en cada uno de los subperíodos analizados, por lo que se hace necesario verificar si desde el punto de vista estadístico existe o no alguna relación entre el nivel de riesgo beta de los activos y carteras con la situación de la economía o momento bursátil, medida a través de la prima de riesgo del mercado. Por ello, y siguiendo a Marín y Rubio (2001:396) vamos a estimar la siguiente regresión lineal, tanto para los títulos individuales como para las carteras sectoriales:

$$
r_{i t}=\alpha_{i 1}+\alpha_{i 2} D_{t}+\beta_{i 1} R_{M t}+\beta_{i 2}\left(D_{t} R_{M t}\right) \varepsilon_{i t} .
$$

Dicha eculación de regresión múltiple recoge la variable ficticia $D_{t}$, la cual toma el valor 1 cuando el mercado bursátil se encuentra en alza, por lo que la prima de riesgo del mismo es positiva, y el valor 0 en caso de que el mercado esté a la baja.

El coeficiente $\beta_{i 2}$ nos indica el efecto diferencia del mercado en alza sobre el riesgo beta. Si dicho coeficiente es estadísticamente igual a cero, el riesgo beta no se ve influenciado por la situación de alza o baja del mercado. En cambio, si tal coeficiente es estadísticamente distinto de cero, se puede aceptar que el riesgo beta es diferente en función de que el mercado se encuentre en alza o a la baja. Además, en esta hipótesis, si toma un valor negativo, se puede afirmar que existe una relación decreciente entre el momento bursátil y el nivel de riesgo 
beta, verificándose, por el contrario, la existencia de una relación creciente, si toma dicho coeficiente un valor positivo.

Tras efectuarse la estimación del coeficiente $\beta_{i 2}$ para cada uno de los títulos individuales, tomando los tres índices bursátiles señalados, los resultados obtenidos se han sintetizado en la Tabla 12. De dicha tabla se desprende que, con los tres índices, la mayoría de los títulos individuales presentan coeficientes $\beta_{i 2}$ estadísticamente nulos, por lo que el comportamiento del riesgo beta de los títulos individuales no se modifica en función de que el mercado bursátil esté en alza o en baja.

Tabla 12: Significatividad del coeficiente $\beta_{i 2}$ para los títulos individuales

\begin{tabular}{c|c|cc}
\hline & & \multicolumn{2}{|c}{$\beta_{i 2} \neq 0$} \\
\hline & $\beta_{i 2}=0$ & $\beta>0$ & $\beta<0$ \\
\hline IGBM & $95 \%$ & $3,3 \%$ & $1,7 \%$ \\
Índice Equiponderado & $78,4 \%$ & $8,3 \%$ & $13,3 \%$ \\
Índice Ponderado & $91,7 \%$ & $3,3 \%$ & $5 \%$ \\
\hline
\end{tabular}

Al tomar el índice equiponderado, el 21,6\% de los activos tienen un coeficiente estadísticamente distinto de cero, por lo que muestran un riesgo beta que depende de la situación económica. Dicho porcentaje disminuye al $8,3 \%$ y al $5 \%$, al utilizar el indice ponderado por capitalización y el IGBM, respectivamente. Los títulos cuyas variaciones en el riesgo beta mantienen una relación positiva con la situación económica, es decir, aquellos activos cuyo riesgo beta tiende a aumentar cuando el mercado se encuentra en situación de alza, disminuyendo en caso contrario, representan el 8,3\% del total al tomar el índice equiponderado, y el $3,3 \%$ al utilizar los otros dos índices. Sin embargo, los restantes títulos, que representan el $13,3 \%$, el $5 \%$ y el $1,7 \%$ tienen un coeficiente negativo al utilizar el índice equiponderado, el ponderado y el IGBM, respectivamente, mostrando una relación negativa entre el riesgo beta y el momento bursátil.

Una vez realizadas las estimaciones del $\beta_{i 2}$ correspondientes a los distintos sectores, se observa que independientemente de la cartera de mercado utilizada, los resultados muestran que en el $90 \%$ de las carteras sectoriales, el mencionado coeficiente es estadísticamente igual a cero, por lo que el riesgo beta no está influenciado por la situación de la economía. En el 10\% restante, dicho riesgo es distinto en función de la situación de alza o baja del mercado bursátil, existiendo una relación positiva entre ambos. Lo anterior se recoge en la Tabla 13.

Tabla 13: Significatividad del coeficiente $\beta_{i 2}$ para las carteras sectoriales

\begin{tabular}{c|c|cc}
\hline & & \multicolumn{2}{|c}{$\beta_{i 2} \neq 0$} \\
\hline & $\beta_{i 2}=0$ & $\beta>0$ & $\beta<0$ \\
\hline IGBM & $90 \%$ & - & $10 \%$ \\
Índice Ponderado & $90 \%$ & - & $10 \%$ \\
\hline
\end{tabular}

De acuerdo con los resultados alcanzados, podemos afirmar que no existe ninguna relación entre el riesgo beta de los activos y carteras las estimaciones bienales 
con respecto a la situación de la economía, ya que en la mayor parte de los títulos y de las carteras sectoriales el riesgo beta no se ve influenciado por la situación de alza o baja del mercado.

\section{Predicción del riesgo beta}

Una vez analizado en los epígrafes precedentes la estabilidad y el comportamiento temporal del riesgo beta en el mercado español, procede investigar la posibilidad de realizar predicciones de dicho riesgo, siendo la proyección futura del mismo una de las principales utilidades de su estimación. Si bien existen distintas metodologías de proyección, nos inclinamos por la utilización de las tres clásicas que consideramos de mayor relevancia: a) la beta según el modelo de mercado o beta tradicional; b) la beta deducida según la metodología propuesta por Blume (1977) y c) la beta obtenida de acuerdo con el método de Vasicek (1973).

En cualquiera de las tres metodologías nos encontramos con dos clases de betas: la predicha para el intervalo futuro considerado y la observada, la cual tomamos como una estimación de la beta verdadera, siendo ésta obtenida con los datos históricos de las rentabilidades correspondientes al período de predicción.

Para aplicar las metodologías expuestas, el intervalo temporal de la muestra 1991-2000, se ha divido en cinco subperíodos de dos años de duración. Los intervalos 1995-1996, 1997-1998 y 1999-2000 son los períodos de predicción, en los que se va a llevar a cabo la estimación de la beta predicha. Disponemos únicamente de tres subperíodos de predicción ya que para la metodología de Blume se precisa de los datos de rentabilidad correspondientes a los dos intervalos inmediatamente anteriores al de predicción. Así mismo, para la metodología de la beta tradicional y de la de Vasicek, es necesario utilizar únicamente las rentabilidades correspondientes al intervalo inmediatamente anterior al de predicción.

De entre las metodologías de predicción señaladas, es preciso seleccionar aquella que proporcione mejores estimaciones, por lo que resulta necesario disponer de alguna medida del error de predicción que, al comparar los resultados obtenidos según los tres métodos citados, nos indique cuál debe elegirse en función de la precisión de las predicciones. Para ello, una vez predicho el coeficiente beta de un activo o cartera, en un determinado período de predicción y según una metodología, es necesario compararlo con el verdadero valor de la beta, obteniéndose el error de predicción, calculado según la expresión:

$$
e_{j h}^{i}=\left(\hat{\beta}_{j h}^{i}-\beta_{j h}\right)
$$

siendo

$e_{j h}^{i}$ :error de predicción del riesgo beta de un activo o cartera $j$ para el período $h$, según la metodología $i$.

$\hat{\beta}_{j h}^{i}$ :beta predicha para un activo o cartera $j$ en el período de predicción $h$ y según la metodología $i$.

$\beta_{j h}$ :beta verdadera u observada del activo o cartera $j$ en el período $h$.

A efectos de deducir la metodología óptima de predicción, es preciso disponer de alguna medida global del conjunto de errores, de tal forma que aquella 
metodología que presente un menor valor de esa medida global, será la que consideraremos óptima para la predicción. Como medida global del error de predicción, vamos a utilizar las siguientes medidas: el error cuadrático medio (ECM), el error absoluto medio (EAM) y el error absoluto en términos relativos(EAM(TR)), efectuándose para cada una de estas tres medidas la comparación por períodos y por activos (o cartera). Las expresiones analíticas correspondientes a cada una de las mencionadas medidas se recogen en la Tabla 14.

Tabla 14: Medidas de la capacidad predictiva de las distintas metodologías

\begin{tabular}{ccc}
\hline Error & Comparación por periodos & Comparación por activos \\
\hline $\begin{array}{c}\text { Cuadrático } \\
\text { Medio }\end{array}$ & $E C M_{h}^{i}=\frac{1}{N} \sum_{j=1}^{N}\left(\hat{\beta}_{j h}^{i}-\beta_{j h}\right)^{2}$ & $E C M_{j}^{i}=\frac{1}{H} \sum_{h=1}^{H}\left(\hat{\beta}_{j h}^{i}-\beta_{j h}\right)^{2}$ \\
\hline $\begin{array}{c}\text { Absoluto } \\
\text { Medio }\end{array}$ & $E A M_{h}^{i}=\frac{1}{N} \sum_{j=1}^{N}\left|\hat{\beta}_{j h}^{i}-\beta_{j h}\right|$ & $E A M_{j}^{i}=\frac{1}{H} \sum_{h=1}^{H}\left|\hat{\beta}_{j h}^{i}-\beta_{j h}\right|$ \\
\hline $\begin{array}{c}\text { Absoluto Medio } \\
\text { en términos } \\
\text { relativos }\end{array}$ & $E A M(T R)_{h}^{i}=\frac{1}{N} \sum_{j=1}^{N}\left|\frac{\beta_{j h}^{i}-\beta_{j h}}{\beta_{j h}}\right|$ & $E A M(T R)_{j}^{i}=\frac{1}{H} \sum_{h=1}^{H}\left|\frac{\hat{\beta}_{j h}^{i}-\beta_{j h}}{\beta_{j h}}\right|$ \\
\hline
\end{tabular}

$N$ : el número total de activos carteras.

$H$ : número total de periodos de predicción.

Si cualquiera de las medidas anteriores se obtiene por períodos, la misma incluye todos los errores de predicción de todos los activos o carteras en el respectivo período. En cambio, si es obtenida por activos o carteras, se tienen en cuenta exclusivamente los errores de dicho activo o cartera para todos los períodos de predicción.

Puesto que las tres medidas del errror expuestas van a tomar siempre valores positivos, aquella metodología que proporcione un error más cercano a cero es la que tiene una mayor capacidad de predicción, por lo que se puede efectuar una comparación entre los distintos métodos de predicción del riesgo beta.

Una vez definidas las distintas medidas del error de predicción, procedemos a explicar con mayor profundidad cada una de las metodologías que nos permite predecir del coeficiente beta.

\section{a) Modelo de mercado}

Este método consiste en estimar el riesgo beta de forma tradicional en el período de predicción mediante la regresión lineal, utilizando los datos de rentabilidad del período de estimación $h-1$. Ambos períodos $h$ y $h-1$ constan de 24 observaciones mensuales. Dicha metodología supone implícitamente que las estimaciones del riesgo beta son constantes a lo largo del tiempo, por lo que las predicciones obtenidas por este método han de presentar un mayor error de predicción en comparación con cualquier otra metodología que introduzca un comportamiento temporal de las betas. 


\section{b) Metodología de estimación de Blume (1977)}

Este método de predicción incorpora la tendencia temporal del riesgo beta a lo largo del horizonte temporal. De esta forma, para estimar el coeficiente beta durante el período de predicción $h$, se lleva a cabo la estimación de las betas correspondientes a los períodos anteriores $h-1$ y $h-2$, puesto que la hipótesis que sustenta este método es que la tendencia entre los períodos $h-2$ y $h-1$, es igual a la tendencia entre los períodos $h-1$ y $h$.

El procedimiento a seguir para determinar la beta predicha para el período $h$ es el siguiente. En primer lugar se llevan a cabo las estimaciones del riesgo beta, para cada uno de los activos o carteras, correspondientes a los períodos $h-$ 2 y $h-1$, que denominamos $\beta_{j h-2}$ y $\beta_{j h-1}$. Todas estas estimaciones se calculan con datos de 24 observaciones mensuales. Una vez realizadas las mencionadas estimaciones, obtenemos dos series de betas estimadas, que corresponden a los períodos $h-2$ y $h-1$, las cuales incluyen el total de los activos o carteras que constituyen la muestra. Posteriormente se realiza la regresión lineal entre las dos series anteriores de betas estimadas, obteniéndose la ordenada en el origen $a_{h}$ y la pendiente de la regresión $b_{h}$ :

$$
\beta_{j h-1}=a_{h}+b_{h} \beta_{j h-2}+\mu_{j h-1} .
$$

Los coeficientes $a_{h}$ y $b_{h}$ estimados recogen la tendencia temporal del coeficiente beta en el intervalo temporal $(h-2, h-1)$ y son utilizados para obtener la predicción del riesgo beta para el período $h$ :

$$
\hat{\beta}_{j h}=a_{h}+b_{h} \beta_{j h-1} .
$$

\section{c) Metodología de estimación de Vasicek (1973)}

Este método considera la tendencia del riesgo beta hacia la beta media, no realizándose el mismo ajuste para todos los activos individuales o carteras, como ocurre en la metodología de Blume(1977), sino que dicho ajuste depende del error de estimación del coeficiente beta respectivo. Cuanto mayor sea la diferencia entre el coeficiente beta de cada activo o cartera con la beta media, mayor será su error de estimación, y por consiguiente, mayor ha de ser el ajuste a realizar.

El proceso a seguir para la obtención de la predicción del riesgo beta correspondiente al período $h$ se describe a continuación. Primeramente es necesario estimar las betas de todos los activos o carteras del periodo $h-1$, las cuales se obtienen utilizando las 24 observaciones de rentabilidad disponibles en las respectivas muestras. En cada una de las estimaciones llevadas a cabo es necesario determinar el cuadrado del error estándar del estimador beta, el cual recoge la varianza estimada del estimador, y que denominamos $\sigma_{\beta_{j h-1}}^{2}$. A continuación y con la series de betas estimadas para el mencionado periodo $h-1,60$ para los activos y 10 para los sectores, se calcula la beta media $\bar{\beta}_{h-1}$ y la cuasivarianza $\sigma_{\bar{\beta}_{h-1}}^{2}$.

La predicción del riesgo beta para el período $h$ se obtiene como la suma ponderada de la beta media y la beta del activo o cartera estimada en el período 
inmediatamente anterior. La expresión que nos permite su cálculo es la siguiente:

$$
\hat{\beta}_{j h}=\frac{\sigma_{\beta_{j h-1}}^{2}}{\sigma_{\beta_{h-1}}^{2}+\sigma_{\beta_{j h-1}}^{2}} \bar{\beta}_{T}+\frac{\sigma_{\bar{\beta}_{h-1}}^{2}}{\sigma_{\bar{\beta}_{h-1}}^{2}+\sigma_{\beta_{j h-1}}^{2}} \beta_{j h-1} .
$$

Dicha expresión pone de manifiesto que el ajuste hacia la media, que se realiza a los coeficientes beta de los activos o carteras que tengan un error de estimación elevado, es superior al correspondiente a aquellos con menores errores de estimación.

Una vez expuestas las tres metodologías, se lleva a cabo las predicciones de las betas de los títulos y de los sectores en los perídos 1995-1996, 1997-1998 y 1999-2000. El análisis comparativo de los distintos metodos de predicción del riesgo beta se va a efectuar mediante las tres medidas de predicción señaladas.

Una síntesis de los resultados obtenidos tras la predicción del riesgo beta, utilizando las tres metodologías y tomando como medida del error el ECM, EAM y el EAM(TR), para la muestra de títulos individuales y de carteras sectoriales, se recoge en las Tablas 15 y 16, respectivamente. En las mencionadas tablas se expone la media de todos los errores de predicción, cuadráticos medios, absolutos medios y absolutos medios en términos relativos. Dichos valores se pueden obtener, bien como la media aritmética de los errores obtenidos en la comparación por activos (o carteras), o bien como la media de los errores correspondientes a la comparación por períodos de predicción, puesto que de cualquiera de las dos formas el valor medio es el mismo.

Tabla 15: Media aritmética del error de predicción (títulos individuales)

\begin{tabular}{|c|c|c|c|}
\hline \multicolumn{4}{|c|}{ Error Cuadrático Medio (ECM) } \\
\hline Método & IGBM & I. Equipond. & I. Ponderado \\
\hline Beta Modelo de Mercado & 0.3949 & 0.3196 & 0.3390 \\
\hline Beta Blume & 0.3038 & 0.2485 & 0.2540 \\
\hline Beta Vasicek & 0.9240 & 0.5864 & 0.9150 \\
\hline \multicolumn{4}{|c|}{ Error Absoluto Medio (EAM) } \\
\hline Periodo & IGBM & I. Equipond. & I. Ponderado \\
\hline Beta Modelo de Mercado & 0.4881 & 0.4356 & 0.4571 \\
\hline Beta Blume & 0.4489 & 0.3869 & 0.4045 \\
\hline Beta Vasicek & 0.8380 & 0.6484 & 0.8333 \\
\hline \multicolumn{4}{|c|}{ Error Absoluto Medio en términos relativos(EAM(TR)) } \\
\hline Periodo & IGBM & I. Equipond. & I. Ponderado \\
\hline Beta Modelo de Mercado & 2,0900 & 0,7371 & 2,2022 \\
\hline Beta Blume & 2,4479 & 0,7875 & 2,6375 \\
\hline Beta Vasicek & 4,2040 & 1,4205 & 4,6882 \\
\hline
\end{tabular}

Al tomar como medida del error de predicción ECM y EAM para los activos considerados individualmente, tal y como se muestra en la Tabla 15, se observa que son siempre más cercanos a cero al utilizar la metodología de Blume, por 
lo que dicho método es el que proporciona una mayor capacidad predictiva. En segundo lugar se encuentra el modelo de mercado, que calcula la beta de forma tradicional, situándose el modelo propuesto por Vasicek como el de peor capacidad predictiva, ya que sus errores son siempre superiores al resto.

Si consideramos el $\operatorname{EAM}(\mathrm{TR})$, una medida que recoge la proporción del error sobre el verdadero valor del coeficiente beta, los resultados difieren en cuanto al mejor método de predicción para los títulos individuales, puesto que ahora es el modelo de mercado, y no la metodología de Blume, que se sitúa en segundo lugar. El método de Vasicek continúa siendo el peor de todos, obteniendo errores que prácticamente duplican a los anteriores. Referente al índice que se toma como proxy de la cartera de mercado, hay que destacar que, independientemente de la metodología y de la medida del error de predicción, al utilizar el índice equiponderado se obtienen los menores errores de predicción.

Tabla 16: Media aritmética del error de predicción (carteras sectoriales)

\begin{tabular}{ccc}
\hline \multicolumn{3}{c}{ Error Cuadrático Medio (ECM) } \\
\hline Método & IGBM & I. Ponderado \\
\hline Beta Modelo de Mercado & 0.1274 & 0.3311 \\
Beta Blume & 0.1098 & 0.2714 \\
Beta Vasicek & 2.4103 & 2.7170 \\
\hline Error Absoluto Medio (EAM) \\
\hline Periodo & IGBM & I. Ponderado \\
\hline Beta Modelo de Mercado & 0.2787 & 0.3624 \\
Beta Blume & 0.2670 & 0.2469 \\
Beta Vasicek & 1.4956 & 1.5977 \\
\hline Error Absoluto Medio en términos relativos(EAM(TR)) \\
\hline Periodo & IGBM & I. Ponderado \\
\hline Beta Modelo de Mercado & 0,6765 & 1,4106 \\
Beta Blume & 0,6299 & 1,2539 \\
Beta Vasicek & 2,9451 & 4,9136 \\
\hline
\end{tabular}

En cuanto a los errores obtenidos con la estimación de la beta en las carteras sectoriales, recogidos en la Tabla 16, hay que señalar que el método de Blume es el que proporciona un menor error de predicción, ya sea medido éste a través del ECM, del EAM o del EAM(TR). El método de Vasicek vuelve a ser el peor método de predicción al presentar los mayores errores, situándose por detrás del modelo de mercado. Al considerar exclusivamente el ECM se observa que los errores de predicción al utilizar el IGBM son siempre inferiores a los correspondientes al índice ponderado por capitalización, independientemente de la metodología utilizada. Al utilizar como medida del error de predicción el EAM y realizar comparaciones entre los dos índices, tenemos que el IGBM es el que ofrece un menor error en la beta tradicional y en el método de Vasicek, mientras que el índice ponderado proporciona un error de predicción menor en la metodología de Blume. Finalmente, y con referencia al EAM(TR), los resultados indican que los errores de predicción son menores al tomar el IGBM como cartera de mercado, independientemente de la metodología utilizada. 
Tras la predicción del riesgo efectuadas por períodos y por activos, podemos afirmar que independientemente del índice de mercado utilizado, el método de Blume es el que presenta una mayor capacidad predictiva. En segundo lugar, se sitúa la beta tradicional del modelo de mercado, obteniendo errores de predicción inferiores al método de Vasicek, que se sitúa como el peor de los tres métodos.

\section{Conclusiones}

Tras realizar un estudio empírico del riesgo beta en el mercado bursátil español, tanto para los títulos considerados invidualmente como para los activos agrupados en carteras sectoriales, disponemos de un mayor conociniento de dicho riesgo, recogiéndose a continuación las conclusiones más relevantes derivadas de nuestra investigación.

El riesgo beta para el conjunto de títulos individuales es estadísticamente significativo, independientemente del índice utilizado como proxy de la cartera de mercado (IGBM, índice equiponderado y ponderado por capitalización). En cambio, no se verifica la existencia de una relación lineal entre la rentabilidad de las carteras sectoriales y la rentabilidad del índice equiponderado. El riesgo beta estimado para cada sector, $\mathrm{y}$ con independencia del índice utilizado, nos indica que los sectores de Construcción, Metal-Mecánica y Nuevas Tecnologías han presentado un carácter agresivo. En cambio, los sectores de Alimentación, Bancos-Finanzas, Cartera-Inversión, Eléctricas, Petroleo-Química y Otras Industrias son defensivos.

El análisis de la estabilidad del riesgo beta correspondiente a los títulos individuales y carteras sectoriales, evidencia que dicho riesgo no permanece constante a lo largo del tiempo, independientemente del índice bursátil utilizado como proxy de la cartera de mercado. Así mismo, se observa que el porcentaje de coeficientes beta estables aumenta al agrupar los activos en carteras.

El análisis del comportamiento temporal del riesgo beta ha mostrado que, al realizar estimaciones quinquenales, el riesgo beta correspondiente al período 1996-2000 ha sufrido una disminución con respecto a los obtenidos en el intervalo 1991-1995, tanto para los activos como para las carteras sectoriales. Además se ha observado que en la mayoría de los títulos y sectores el carácter del riesgo beta, agresivo o defensivo, es irregular. No obstante, el carácter agresivo o defensivo de un cierto porcentaje de activos y sectores se ha mantenido inalterable en los distintos subintervalos temporales. También se aprecia que la proporción de títulos y sectores con un comportamiento regular, en el carácter del riesgo beta, es superior cuando se toma como cartera de mercado el índice equiponderado y el IGBM, respectivamente.

Al realizar estimaciones bienales, y comparar el coeficiente beta entre dos periodos consecutivos, se pone de manifiesto la existencia de activos y de sectores que presentan variaciones en su riesgo beta similar a la experimentada por la prima de riesgo de mercado. Sin enbargo al realizarse un estudio sobre la existencia de alguna relación entre el riesgo beta de los activos y carteras las estimaciones bienales con respecto a la situación de la economía, los resultados muestran que en la mayor parte de los títulos y de los sectores, el riesgo beta no se ve influenciado por la situación de alza o baja del mercado. 
La predicción del riesgo beta efectuada en los períodos 1995-1996, 19971998 y 1999-2000, ha mostrado que la metodología propuesta por Blume (1977) es la que ofrece mejores predicciones al proporcionar una superior capacidad predictiva. En cambio, el método de Vasicek (1973) es el que tiene los errores de predicción más elevados. Además, la utilización de distintos índices bursátiles como aproximación de la cartera de mercado nos indica que el equiponderado para los activos individuales y el IGBM para las carteras sectoriales, son los que proporcionan resultados más satisfactorios en la estimación futura del riesgo beta, ya que si utilizamos los mencionados índices se obtienen los menores errores de predicción, con independencia de la metodología y medida del error utilizada.

\section{Anexo}

Tabla I: Muestra de títulos individuales

\begin{tabular}{|c|c|c|}
\hline $\mathrm{N}^{\circ}$ & Sigla & Títulas \\
\hline 1 & $\mathrm{ACE}$ & Autopistas Concesionaria Española \\
\hline 2 & ACS & Actividades de Construcción y Servicios \\
\hline 3 & $\mathrm{ACX}$ & Acerinox \\
\hline 4 & AGS & Aguas Barna \\
\hline 5 & ALB & Corporación Financiera Alba \\
\hline 6 & ALT & Altadis \\
\hline 7 & AMP & Amper \\
\hline 8 & ANA & Acciona \\
\hline 9 & AND & Banco de Andalucía \\
\hline 10 & ASA & Tavex Algodonera \\
\hline 11 & ATL & Banco Atlántico \\
\hline 12 & AUM & Aurea Concesiones \\
\hline 13 & $\mathrm{AZC}$ & Asturiana del Zinc \\
\hline 14 & AZK & Azkoyen \\
\hline 15 & $\mathrm{BAM}$ & Inmobiliaria Bami \\
\hline 16 & BBVA & Banco Bilbao Vizcaya Argentaria \\
\hline 17 & $\mathrm{BKT}$ & Bankinter \\
\hline 18 & BVA & Banco deValencia \\
\hline 19 & CAN & Hidroeléctrica del Cantábrico \\
\hline 20 & CAS & Banco de Castilla \\
\hline 21 & CBL & Banco de Crédito Balear \\
\hline 22 & CEP & Cepsa \\
\hline 23 & CPF & Campofrío \\
\hline 24 & CRI & Saint Gobain Cristalería \\
\hline 25 & CTG & Gas Natural \\
\hline 26 & $\mathrm{DRC}$ & Dragados \\
\hline 27 & ECR & Ercrós \\
\hline 28 & ELE & Endesa \\
\hline 29 & $\mathrm{ENC}$ & Grupo Empresarial Ence \\
\hline 30 & FCC & Fomento de Construcciones y Contratas \\
\hline
\end{tabular}


Tabla I: (continuación)

\begin{tabular}{ccc}
\hline $\mathrm{N}^{\circ}$ & Sigla & Títulos \\
\hline 31 & FIL & Filo \\
32 & GAL & Banco de Galicia \\
33 & HKN & Heineken \\
34 & IBE & Iberdrola \\
35 & KOI & Koipe \\
36 & MAP & Corporación Mapfre \\
37 & MDF & Grupo Duro Felguera \\
38 & MVC & Metrovacesa \\
39 & NMQ & N. Montaña \\
40 & PAS & Banco Pastor \\
41 & POP & Banco Popular Español \\
42 & PSG & Prosegur \\
43 & PUL & Puleva \\
44 & REP & Repsol \\
45 & SCH & Baṇco Santander Central Hispano \\
46 & SNC & Sniace \\
47 & STG & Sotogrande \\
48 & TEF & Telefónica \\
49 & TFI & Tafisa \\
50 & TUB & Tubacex \\
51 & TUD & Tudor \\
52 & UNF & Unión Fenosa \\
53 & URA & Uralita \\
54 & URB & Urbis \\
55 & VAL & Vallehermoso \\
56 & VAS & Banco de Vasconia \\
57 & VDR & Portland Valderrivas \\
58 & VIS & Viscofán \\
59 & ZOT & Zardoya Otis \\
60 & ZRG & Banco Zaragozano \\
\hline & &
\end{tabular}

\section{Bibliografía}

Blume, M. (1977). Betas and their Regression Tendencies. In Friend I. and J. Bicksler (Eds.). Risk and Return in Finance. Balliriger Publishing Company, Cambridge.

Chow, G. (1960). Test of Equality between sets of Coefficients in two Linear Regressions. Econometrica, 28, pp. 591-605.

Durbin, J. y G.S. Watson (1972). Contraste de correlación serial en la regresión minimocuadrática. En Alcaide, A. (1972). Lecturas de Econometría. Madrid. Ed. Gredos, pp. 164-186.

Faff, R. and R. Brooks (1997). Further Evidence on the Relationship between Beta Stability and the Length of the Estimation Period. Advances in Investment Analysis and Portfolio Management, 4, pp. 95-111. 
Gómez-Bezares, F., J. A. Madariaga y J. Santibañez (1994). Valoración de acciones en la Bolsa Española. Biblioteca de Gestión. Ed. Desclée de Brouwer. Bilbao.

Lintner, J. (1965). The Valuation of Risk Assets and the Selection of Risky Investments in Stock Portfolios and Capital Budgets. The Review of Economics and Statistics, 4, pp. 13-37.

Markowitz, H. (1952). Portfolio Selection. The Journal of Finance, 7, pp. 77-91.

Marín, J. M. y G. Rubio (2001). Economía Financiera. Ed. Antonio Bosch. Barcelona.

Mossin, J. (1966). Equilibrium in a Capital Asset Market. Econometrica, 34, pp. 768-783.

Russell, G., M. Impson, and I. Karafiath (1994). An Empirical Investigation of Beta Stability: Portfolios vs. Individual Securities. Jourmal of Business and Acconnting, 21(6), pp. 909-917.

Sharpe, W. F. (1963). A Simplified Model for Portfolio Analysis. Management Science, pp. 277-293.

Sharpe, W.F. (1964). Capital Asset Prices: a Theory of Market Equilibrium under Conditions of Risk. The Journal of Finance, 19(3), pp. 425-442.

White, H. (1980). A Heteroscedasticity-consistent Covariance Matriz Estimator and a Direct Test of Heteroscedasticity. Econometrica, 48, pp. 817-838.

Vasicek, O. (1973). A Note on Using Cross-Sectional Information in Bayesian Estimation of Security Betas. Journal of Finance, 28, pp. 1233-1239. 